\title{
Les rayonnements ionisants Applications médicales et industrielles*
}

\author{
H. VIDAL**
}

(Manuscrit reçu le 8 octobre 1993)

RÉSUMÉ La radiographie $X$ utilisée pour le diagnostic médical est l'application la plus connue des rayonnements ionisants parce que la plus répandue avec, toutes catégories confondues, près de 57500 installations en France. D'autres applications médicales, concernant un nombre d'installations beaucoup plus limité, bénéficient, elles aussi, d'une grande notoriété et font appel aux radioéléments artificiels : la télégammathérapie (167 installations), la curiethérapie (119 installations) ou la thérapie à l'aide de sources non scellées (257 installations). Les applications industrielles dont la diversité est considérable sont beaucoup moins connues. Si l'on connaît assez bien l'usage des rayons $X$ ou gamma pour les contrôles non destructifs, l'irradiation industrielle de certains aliments ou l'usage des traceurs radioactifs, on ignore généralement que des sources radioactives sont utilisées pour la mesure de paramètres intervenant dans le contrôle de nombreux procédés industriels. Le nombre de titulaires d'autorisations de détenir, d'utiliser et/ou de céder des radioéléments artificiels est au total d'environ 4800 dont près de 650 en médecine et 500 en recherche médicale. Sans prétendre à l'exhaustivité, l'article passe en revue quelques applications typiques des rayonnements ionisants, tant dans le domaine médical qu'industriel. De nombreuses indications chiffrées sont données sur ces techniques, qu'elles concernent le nombre d'utilisateurs autorisés ou les quantités de radioéléments mis en cuvre.

ABSTRACT Medical diagnosis with X-rays is the best known use of ionizing radiations on account of its wide diffusion (about 57500 units in France). Other medical applications of artificial radionuclides involving a smaller number of installations are also well known, i.e. gamma teletherapy (167 units), brachytherapy (119 units) or therapy using unsealed sources (257 units). The industrial uses of ionising radiation, the diversity of which is vey large, are generally less well known. The use of $X$ - and gamma rays for non-destructive testing or food preservation and the use of tracers have some notoriety, but few people know that radioactive sources are involved in the measurement of parameters controlling industrial processes. The number. of persons authorized to hold, use and/or sell artificial radionuclides amounts to about 4800 , all applications included. Approximately 650 of them are involved in therapy and 500 in medical research. The aim of this paper, which is not exhaustive, is to review a few typical applications of radionuclides both in the medical and industrial fields. It also supplies data both on the number of people authorized to use each technique and the radionuclides involved.

\footnotetext{
* Communication présentée lors des journées SFRP "Sécurité des sources et gênérateurs de rayonnements ionisants", Saclay, 9-10 juin 1993.

** Commission interministérielle des radioéléments artificiels (CIREA), Secrétariat permanent, BP 90, 92260 Fontenay-aux-Roses. Adresse actuelle : Direction du Centre d'Etudes nucléaires de Fontenay-aux-Roses, BP 6, 92660 Fontenay-aux-Roses Cedex.
} 


\section{Introduction}

Les applications industrielles ou médicales des rayonnements ionisants se sont développées de façon très importante durant ces vingt dernières années. Pour illustrer ce propos, on peut noter (Fig. 1) que le nombre de personnes autorisées à détenir des radioéléments artificiels a pratiquement doublé pendant cette période et que le nombre de demandes de fournitures de radioéléments sous forme de sources non scellées a été multiplié par 5 . Ces dernières années, le nombre d'utilisateurs est voisin de 5000 et le nombre de livraisons se situe autour de 200000 par an. Les applications les plus nombreuses et les plus connues restent, cependant, liées aux utilisations des rayons $\mathrm{X}$ dans le domaine du radiodiagnostic médical et dentaire avec plus de 56000 installations.

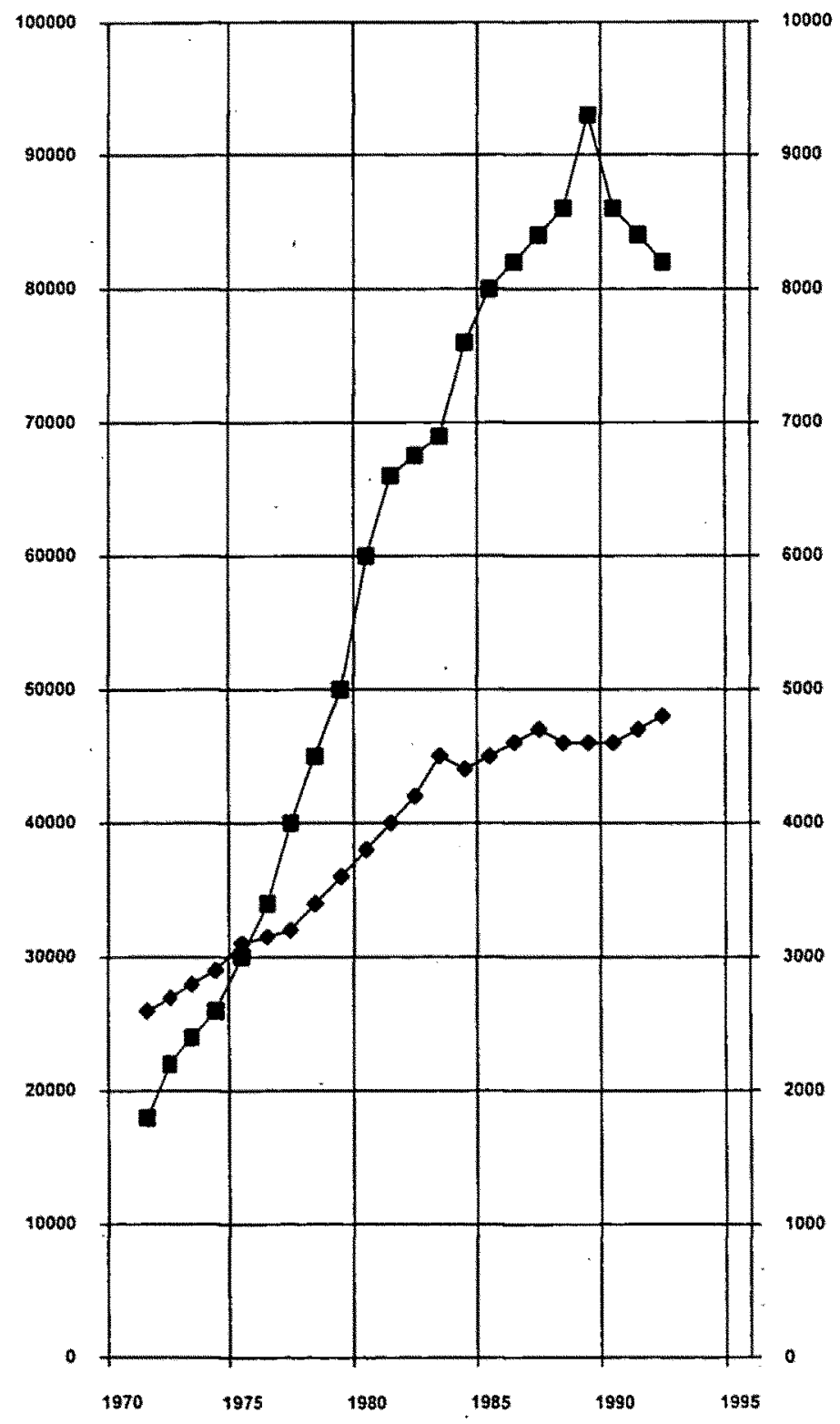

Fig. 1. - Utilisation des radioéléments artificiels.

- Demandes de fournitures sources non scellées,

- Personnes autorisées

Uses of articificial radionuclides 
Cet article se propose d'une part de présenter les derniers éléments statistiques (établis en 1992 ou 1993) concernant l'utilisation des sources de rayonnements ionisants en France, d'autre part, de décrire quelques exemples d'utilisations parmi les moins connues.

\section{Applications médicales des rayonnements ionisants}

Les praticiens et établissements, utilisant à des fins thérapeutiques ou de diagnostic des appareils générateurs de rayonnements ionisants ou des radioéléments, ne peuvent procéder à des radiodiagnostics concernant des assurés sociaux ou leur dispenser des soins que s'ils ont obtenu l'agrément de chacun des appareils et installations qu'ils détiennent à cet usage. Ces appareils et installations sont classés dans diverses catégories en fonction de leurs applications. Suivant les catégories, les agréments sont donnés par le ministre chargé de la santé ou par le préfet après avis du Service central de protection contre les rayonnements ionisants (SCPRI).

\subsection{Radiodiagnostic médical et dentaire}

Les opérations de radiodiagnostic médical et dentaire sont effectuées aux moyens de générateurs de rayons $X$. La classification des installations est donnée au tableau I et le nombre de celles qui sont agréées en 1993 est indiqué au tableau II.

TABLEAU I

Classement des installations radiologiques médicales Classification of radiological installations for medical use

\begin{tabular}{|l|lll|}
\hline & A & Radioscopie en cardiologie, pneumophtisiologie \\
B & Membres, parties molles (sauf mammo.) \\
C & B + organes thoraciques, tout squelette \\
D & Tous actes de radiodiagnostic \\
Générateurs & E & $\begin{array}{l}\text { Radio dentaire } \\
\text { électriques }\end{array}$ \\
F & Radiophotographie \\
M & Tomographie X \\
N & Mammographie \\
\hline G & Traitement superficiels (X) \\
H & Radiothérapie à haute énergie (accélérateurs) \\
Radioéléments & J & Télégammathérapie \\
artificiels & Curiethérapie \\
& L & Médecine nucléaire (sources non scellées) \\
\hline
\end{tabular}

La catégorie A concerne les installations fixes, destinées à la radioscopie, utilisées par un médecin qualifié en électroradiologie, cardiologie ou pneumophtisiologie. 
TABLEAU II

Radiodiagnostic médical et dentaire - Rayons $X$ Medical and dental radiodiagnosis - $\mathbf{X}$-rays

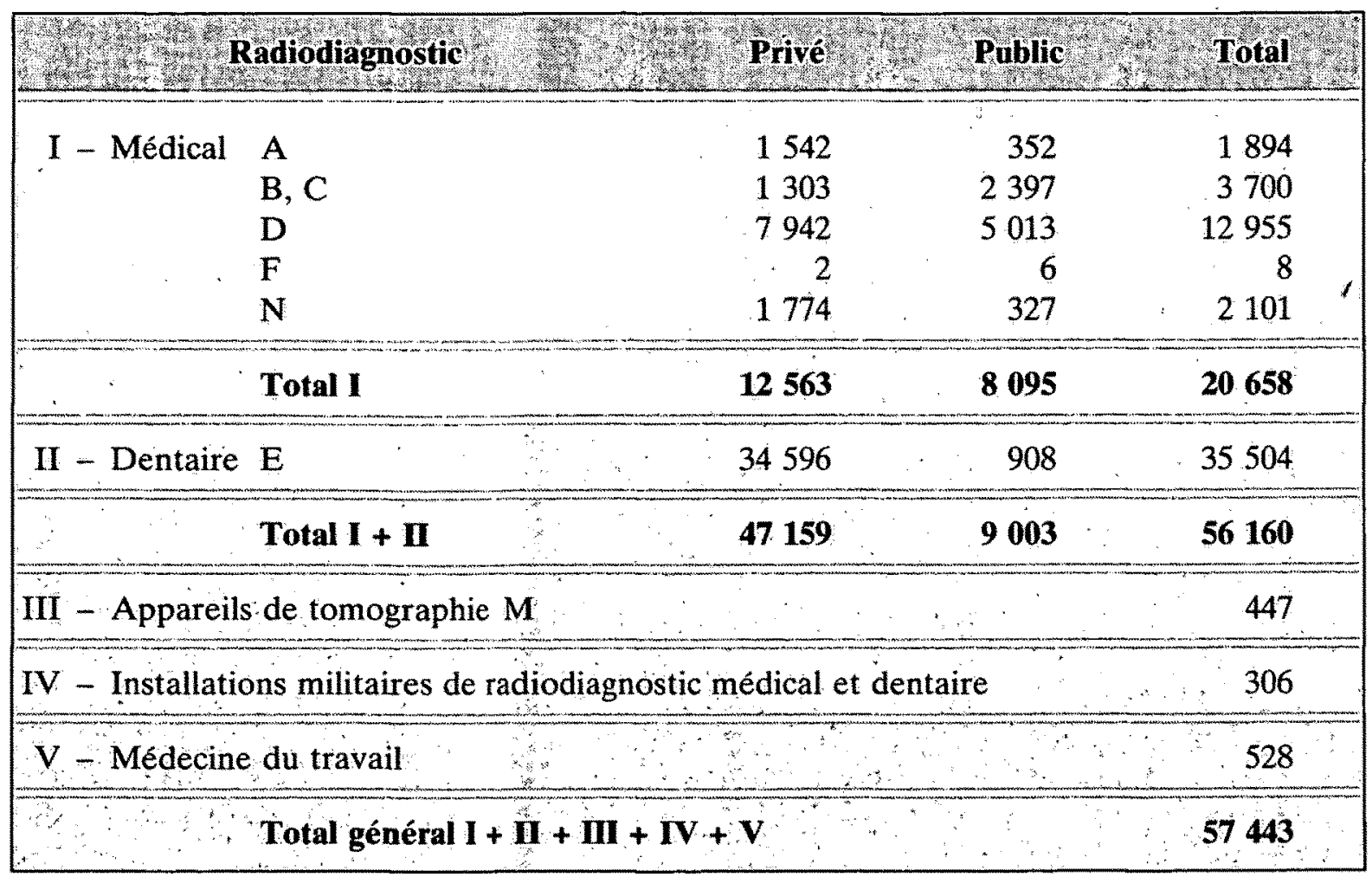

Les installations de la catégorie B sont équipées d'appareils pèrmettant d'obtenir une intensité d'au moins $30 \mathrm{~mA}$ sous une tension minimale de $105 \mathrm{kV}$ destinées à la radiographie des membres et des parties molles, à l'exclusion de la mammographie.

La catégorie $C$ couvre les installations dont les appareils peuvent délivrer une intensité de $100 \mathrm{~mA}$ sous une tension d'au moins $105 \mathrm{kV}$. Elles permettent de couvrir les actes de la catégorie $\mathrm{B}$ outre la radiographie des organes thoraciques et de l'ensemble du squelette.

La catégorie $D$ concerne les installations permettant d'effectuer l'ensemble des actes de radiodiagnostic. Les appareils doivent pouvoir délivrer $200 \mathrm{~mA}$ sous $105 \mathrm{kV}$.

Les autres catégories sont :

- E pour le radiodiagnostic dentaire,

- F pour la radiophotographie qui ne représente que quelques installations,

- M pour la tomographie $\mathrm{X}$ et,

- N pour la mammographie.

Enfin, il faut noter que les appareils de radiographie destinés à la médecine préventive du travail sont soumis seulement à déclaration. 


\subsection{Générateurs électriques de radiothérapie}

Les générateurs électriques de radiothérapie sont classés dans les catégories $\mathrm{G}$ et $\mathrm{H}$ (Tab. III).

TABLEAU III

Générateurs de radiothérapie

Radiotherapy generators

\begin{tabular}{|c|c|c|c|}
\hline$G$ & Rayons X & $6 \mathrm{kV}<T<600 \mathrm{kV}$ & 0 \\
\hline $\mathbf{H}$ & Accélérateurs de particules & $E>1 \mathrm{MeV}$ & 192 \\
\hline
\end{tabular}

La catégorie $\mathrm{G}$ concerne les installations équipées d'appareils de rayons $\mathrm{X}$; de tension d'emploi de $6 \mathrm{kV}$ à $600 \mathrm{kV}$, utilisés pour la thérapie superficielle ou de faible profondeur. Quelques installations existent encore mais, datant de plus de 25 ans, aucune n'est homologuée.

La catégorie $\mathrm{H}$ concerne les accélérateurs de particules d'énergie supérieure à $1 \mathrm{MeV}$.

\subsection{Utilisation de radioéléments en sources scellées}

Les applications médicales des radioéléments artificiels sont la télégammathérapie, la curiethérapie et la médecine nucléaire.

La télégammathérapie (catégorie $J$ ) ne peut être effectuée qu'avec des sources scellées de radioéléments d'activité minimale de $18,5 \mathrm{TBq}(500 \mathrm{Ci})$ dont l'énergie est supérieure à $500 \mathrm{keV}$. Les installations agréées, au nombre de 167 , sont équipées d'appareils au cobalt 60 , leur protection biologique étant adaptée à l'activité de la source. La source en service la plus puissante a une activité de $240 \mathrm{TBq}(6500 \mathrm{Ci})$, l'activité moyenne se situant autour de $111 \mathrm{TBq}$ (3000 Ci).

Pour le traitement par curiethérapie (catégorie K) la source radioactive est mise au contact de la tumeur à traiter. Le positionnement des sources pour les irradiations internes est fait à l'aide de cathéters mis en place soit par les voies naturelles soit chirurgicalement. La technique classique met en cuvre des sources de césium 137 ou des fils d'iridium 192 qui délivrent des "bas" ou "moyens" débits de dose. Les sources de césium 137 utilisées pour les "moyens débits" ont une activité se situant autour de $4 \mathrm{GBq}(110 \mathrm{mCi})$. Une nouvelle technique dite à "haut débit" se met en place après avoir été expérimentée ces trois dernières années. Elle utilise des sources d'iridium 192 d'une activité d'environ $370 \mathrm{GBq}(10 \mathrm{Ci})$. 


\subsection{Sources non scellées. Diagnostic in vitro}

Le marquage de substances par l'iode 125 ou le tritium est très utilisé pour les dosages radioimmunologiques. La technique la plus répandue est la technique dite "en sandwich". Elle convient particulièrement aux substances de nature protéinique qui possèdent au moins deux sites antigéniques. Pour réaliser le dosage, on fixe d'abord l'anticorps sur une phase solide, la paroi d'un tube. L'antigène, présent dans le milieu que l'on introduit dans le tube, se fixe sur l'anticorps. On introduit alors un deuxième anticorps marqué à l'iode 125 ou au tritium et on compte la quantité fixée.

Le nombre d'installations autorisées à réaliser des diagnostics in vitro est de 202 (Tab. IV).

\subsection{Irradiation des produits sanguins}

L'irradiation des produits sanguins est une méthode simple d'inhiber la division lymphocytaire afin d'éliminer le risque de maladie post-transfusionnelle de greffon contre l'hôte chez les patients immuno-déprimés. Les irradiateúrs sont des appareils compacts autoprotégés dans lesquels se trouvent une chambre d'irradiation et des sources de cobalt 60 ou de césium 137. Les activités, suivant les appareils, s'étagent de $37 \mathrm{TBq}(1000 \mathrm{Ci})$ à $222 \mathrm{TBq}(6000 \mathrm{Ci})$ environ.

Le nombre d'installations de ce type en France est de 28.

\section{Applications industrielles}

Les générateurs de rayons $\mathrm{X}$, bien que largement utilisés dans le secteur industriel pour réaliser des contrôles non destructifs, ne représentent pas, comme dans le domaine médical, une part prépondérante des sources de rayonnement utilisées. En effet, on ne compte que 735 appareils déclarés alors que les gammagraphes, leur concurrents directs, sont au nombre de 850 et que, toutes spécialités confondues, les industriels utilisateurs de radioéléments artificiels sont plus de 3600 . Les accélérateurs industriels restent assez marginaux en nombre avec 16 installations déclarées.

Les techniques mettant en œuvre les générateurs de rayons X, les appareils de gammagraphie ou les irradiateurs gamma industriels ne seront pas décrites ici car elles sont suffisamment connues. Rappelons, cependant, leurs principales utilisations :

- les générateurs $X$ et les gammagraphes sont utilisés pour les contrôles non-destructifs de pièces, de structures soudées ou d'ouvrages d'art ;

- les accélérateurs sont utilisés en particulier pour la stérilisation de produits médicaux, la modification de propriétés de polymères, l'initiation de la polymérisation de résines, la stérilisation des aliments ; 
- les irradiateurs gamma sont essentiellement utilisés pour la conservation des aliments.

Les autres applications mettant en jeu des radioéléments artificiels sont très nombreuses, aussi quelques exemples seulement en seront donnés ici.

\subsection{Mesures d'épaisseur}

Les mesures d'épaisseur sont réalisées à l'aide de matériels pouvant faire appel, suivant les applications, à deux techniques différentes :

- la transmission bêta ou gamma,

- la rétrodiffusion bêta ou gamma.

Dans le premier cas, la source et le détecteur sont de part et d'autre de la pièce à contrôler, dans l'autre ils sont du même côté. La mesure par transmission est la plus couramment utilisée. L'autre n'est généralement utilisée que lorsqu'il y a difficulté d'accès des deux côtés de l'échantillon ou qu'il s'agit de mesures d'épaisseurs de dépôts. Les radioéléments les plus fréquemment utilisés sont le cobalt 60 , le krypton 85 , le strontium 90 , le césium 137 , le prométhéum 147 et l'américium 241. Les activités peuvent varier de $1 \mathrm{GBq}$ à quelques centaines de $\mathrm{GBq}$, soit de quelques dizaines de millicuries à quelques curies.

Le domaine d'application de chacun de ces radioéléments varie en fonction de la densité surfacique du matériau à mesurer :

- prométhéum 147 : 1 à $15 \mathrm{mg} \cdot \mathrm{cm}^{-2}$

- krypton $85 \quad: 5$ à $100 \mathrm{mg} \cdot \mathrm{cm}^{-2}$

- strontium $90 \quad: 25$ à $500 \mathrm{mg} \cdot \mathrm{cm}^{-2}$

- américium 241 : $1 \mathrm{à} 10 \mathrm{~g} \cdot \mathrm{cm}^{-2}$

- césium $137 \quad: \quad 1$ à $100 \mathrm{~g} \cdot \mathrm{cm}^{-2}$

- cobalt $60 \quad: 10$ à $1000 \mathrm{~g} \cdot \mathrm{cm}^{-2}$

Dés variantes de ces techniques permettent de mesurer des densités de matériaux ou de réaliser des pesages de matériaux transportés sur des convoyeurs ou dans des tuyauteries alimentant des usines d'élaboration de produits divers dans l'industrie chimique, la sidérurgie ou les cimenteries.

\subsection{Mesures de niveau}

Une source de rayonnement gamma et un détecteur sont disposés de part et d'autre du récipient dont on veut contrôler le niveau. L'intensité du rayonnement reçu par le détecteur diminue lorsque le contenu du récipient est situé entre la source et le détecteur, ce qui permet de déclencher les actions souhaitées, par exemple une alarme, l'arrêt du remplissage ou le déclenchement d'un processus de fabrication. Des systèmes plus élaborés permettent de réaliser des asservissements ou des alarmes de niveau haut et de niveau bas. 
Les mesures de niveaux faisant intervenir des sources radioactives sont utilisées dans la mise en cuvre de processus très variés lorsque :

- une grande précision dans la mesure du niveau est recherchée (embouteillage de boissons, de parfums) ;

- les récipients sont opaques (industrie chimique, remplissage de réservoirs de gaz, remplissage de boites métalliques avec des boissons...) ;

- les niveaux sont difficiles à apprécier par des moyens classiques (trémies de matières solides).

Les radioéléments les plus couramment utilisés pour ces applications sont le cobalt 60 et le césium 137 pour les matières denses et les fortes épaisseurs et l'américium 241 pour les embouteillages et les mises en boite de liquide. Les activités vont de $1 \mathrm{GBq}(27 \mathrm{mCi})$ d'américium 241 à quelques centaines de GBq (quelques curies) de cobalt 60 .

\subsection{Mesure d'humidité et de densité des sols}

Des sources d'américium-béryllium, émettrices de neutrons, et des sources de césium 137, émettrices de rayons gamma, sont utilisées pour déterminer la nature des sols en génie civil ou des sous-sols dans les activités de prospection. Les neutrons émis par les sources Am-Be sont ralentis par les collisions avec les atomes légers présents dans le milieu, en particulier l'hydrogène. Leur détection permet de déterminer la présence d'eau ou d'hydrocarbures. La détection des rayonnements gamma émis par le césium permet de déterminer la densité du sol ou des roches. Les activités des sources de césium varient entre $1 \mathrm{GBq}$ et quelques dizaines de $\mathrm{GBq}$ (quelques dizaines et quelques centaines de millicuries) et celle de sources d'américium entre $1 \mathrm{GBq}$ et quelques TBq (quelques dizaines de millicuries et quelques dizaines de curies).

\subsection{Analyseurs d'alliage par fluorescence $X$}

Les analyseurs par fluorescence $X$ sont très utilisés sur parc pour déterminer rapidement la composition des alliages et en faire le tri. Ils sont aussi utilisés en laboratoire pour le dosage d'échantillons, en particulier la recherche des impuretés, étant donné la grande sensibilité et la grande précision des appareils lorsqu'ils sont adaptés à un problème particulier. Ces appareils sont basés sur la détection des raies $\mathrm{X}$ caractéristiques des atomes dont la fluorescence est excitée par des photons de basse énergie. Les énergies des raies $\mathrm{X}$ détectées indiquent donc les éléments présents dans l'échantillon analysé tandis que les hauteurs des pics donnent les quantités présentes.

Les radioéléments les plus utilisés sont le fer 55 (47\% du parc), le cadmium $109(33 \%)$. Le plutonium 238, l'américium 241 et le curium 244 ne représentent ensemble que $20 \%$ du parc. L'activité maximale des sources'est de $3,7 \mathrm{GBq}(100 \mathrm{mCi})$. 


\subsection{Détecteurs à capture d'électrons}

Les détecteurs à capture d'électrons sont abondamment utilisés en chromatographie en phase gazeuse. Le gaz provenant du chromatographe passe dans une chambre d'ionisation qui contient une source de nickel 63 ou de tritium, émetteurs de rayons bêta. Lorsqu'un composant ayant une forte affinité pour les électrons passe dans la chambre, le courant d'ionisation chute. L'activité maximale des sources utilisées est de l'ordre de $500 \mathrm{MBq}(15 \mathrm{mCi}$ environ) pour le nickel 63 et de $7,4 \mathrm{GBq}(200 \mathrm{mCi})$ pour le tritium.

\subsection{Utilisations diverses de radionucléides en sources scellées}

Quelques applications - comme les détecteurs de fumée ou les tubes parasurtenseurs - sont très répandues puisqu'elles concernent plusieurs centaines de milliers d'appareils vendus par an. Les détecteurs de fumée sont équipés d'une source d'américium 241 d'environ $37 \mathrm{kBq}(1 \mu \mathrm{Ci})$, qui est inoffensive, dans un appareil placé au plafond d'une pièce. Toutefois, afin de garder ces appareils sous contrôle, la vente en est interdite au grand public. Les tubes parasurtenseurs, ou tubes à décharge, contiennent principalement des sources de tritium, prométhéum 147 ou cobalt 60 . Ces tubes sont cédés librement sous réserve que leur activité soit inférieure à $37 \mathrm{kBq}(1 \mu \mathrm{Ci})$ pour le cobalt 60 , $370 \mathrm{kBq}(10 \mu \mathrm{Ci})$ pour le prométhéum 147 et $9250 \mathrm{kBq}(250 \mu \mathrm{Ci})$ pour le tritium.

Pour clore cette rubrique "divers" on doit signaler des applications toujours autorisées, mais dont la mise en service de nouveaux appareils est interdite. Il s'agit des paratonnerres équipés de sources radioactives, radium ou américium 241 , et des stimulateurs cardiaques contenant une source de plutonium 238 . Ces interdictions ont été prononcées en application du "principe de justification" qui indique que les risques encourus par l'utilisation de la radioactivité doivent être largement compensés par les avantages procurés. Or, l'efficacité des paratonnerres radioactifs n'a jamais pu être démontrée et les piles "classiques" des stimulateurs cardiaques ont fait tellement de progrès que l'utilisation du plutonium n'est plus justifiée.

\subsection{Utilisation de radionucléides en sources non scellées}

L'utilisation des sources non scellées dans le domaine non médical est excessivement variée tant en recherche que dans les applications industrielles ou des sciences de la terre. Utilisées comme marqueurs, elles permettent de réaliser aussi bien des études d'hydrologie, des contrôles de procédés dans l'industrie chimique, des suivis d'usure de pièces mécaniques ou des recherches de fuites de canalisations. Ces exemples montrent la diversité des applications dont il est clair que les seules limites sont l'imagination des 676 utilisateurs actuellement autorisés, et les contraintes imposées par la CIREA. 


\subsection{Répartition par type d'appareils (hors sources non scellées)}

Le tableau $\mathrm{V}$ donne la répartition par type d'appareils utilisés dans le domaine non médical, c'est-à-dire hors application à l'homme. Les utilisations de sources non scellées pour des opérations de marquage sont trop diverses pour que l'on puisse aisément les classer en quelques rubriques représentatives. En outre, elles n'utilisent généralement pas des installations ou des outils spécifiques.

TABLEAU V

Répartition par type d'appareils (non médical)

Non-medical uses - number of devices of each type

\begin{tabular}{|lr|}
\hline Rayons X industrie & 785 \\
Rayons X vétérinaire & 33 \\
Accélérateurs & 16 \\
\hline Irradiateurs (hors INB - Hors poche de sang) & 80 \\
Irradiateurs (INB) & 5 \\
Gammagraphes & 850 \\
Densité, pesage, niveau & 7700 \\
Epaisseur & 2700 \\
Niveau d'embouteillage & 260 \\
Niveau de gaz & 180 \\
Humidimètre, densimètre (chantiers) & 1100 \\
Analyseurs d'alliage (Fluorescence X) & 200 \\
Détecteurs à capture d'électrons & 1400 \\
\hline
\end{tabular}

En ce qui concerne les appareils utilisant des sources scellées, il est clair que le classement en certaines catégories peut avoir parfois un caractère quelque peu arbitraire. Il s'agit, en particulier, des appareils qui permettent de réaliser des mesures de densité, pesage ou épaisseurs, car ils ont souvent les mêmes caractéristiques.

\section{Eléments statistiques sur l'utilisation des radioéléments artificiels}

Les textes réglementaires régissant l'utilisation des radioéléments artificiels prévoient une procédure d'autorisation différente suivant qu'il s'agit d'application à l'homme et à la biologie humaine ou qu'il s'agit d'autres applications. Les autorisations sont délivrées dans le premier cas par le ministre chargé de la santé après avis de la première section de la Commission interministérielle des radioéléments artificiels (CIREA) et dans le deuxième cas par le président de la Commission après avis de la deuxième section. 
Le tableau VI donne la répartition des autorisations par section et le domaine d'application en sources scellées ou non scellées. Le nombre d'autorisations réparties par types d'utilisations est donné dans le tableau VII. Les évolutions au cours du temps du nombre de sources scellées distribuées, ainsi que de leur activité totale en fonction de leur utilisation sont données dans le tableau VIII.

TABLEAU VI

Répartition des autorisations CIREA et domaines d'application Distribution of CIREA authorizations by utilization areas and types of sources

Répartition des autorisations

\begin{tabular}{|c|c|}
\hline $\begin{array}{c}\text { Sections } \\
\text { 1re section médicale }\end{array}$ & ( \\
\hline 1 re section recherche & 646 \\
\hline $2^{\mathrm{e}}$ section industrielle & 491 \\
\hline Total & 3666 \\
\hline
\end{tabular}

Domaine d'application

\begin{tabular}{|c|c|c|c|}
\hline Sections & cellées & scell & ters \\
\hline 1re section médicale & 388 & 224 & 34 \\
\hline 1re section recherche & 114 & 323 & 54 \\
\hline $2^{\mathrm{e}}$ section industrielle & 2990 & 360 & 316 \\
\hline Total & 3492 & 907 & 404 \\
\hline
\end{tabular}

La consommation des radioéléments dans le domaine médical (thérapeutique et diagnostique) est donnée dans le tableau IX alors que le total des consommations, toutes utilisations confondues, est donné dans le tableau $\mathrm{X}$. 
TABLEAU VII

Principaux utilisateurs de radioéléments, année 1992 Main users of radionuclides, 1992

\begin{tabular}{|c|c|}
\hline Utilisations (en sources non scellées) & Utilisateurs \\
\hline \multicolumn{2}{|l|}{$1^{\text {re }}$ section (médicale et recherche) } \\
\hline Thérapeutique & 111 \\
\hline Diagnostic in vivo & 170 \\
\hline Diagnostic in vitro & 200 \\
\hline Curiethérapie interstitielle & 118 \\
\hline Recherche & 629 \\
\hline Etalonnage et enseignement & 67 \\
\hline \multicolumn{2}{|l|}{$2^{\mathrm{e}}$ section (industrielle) } \\
\hline Recherche & 603 \\
\hline Etalonnage et enseignement & 107 \\
\hline \multicolumn{2}{|l|}{ Utilisations (en sources scellées) } \\
\hline \multicolumn{2}{|l|}{$1^{\text {re }}$ section (médicale et recherche) } \\
\hline Télégammathérapie & 193 \\
\hline Curiethérapie & 127 \\
\hline Ostéodensimétrie & 41 \\
\hline Irradiation de poches de sang & 28 \\
\hline Autres irradiateurs & 20 \\
\hline Marquage anatomique & 121 \\
\hline Chromatographie en phase gazeuse & 126 \\
\hline Etalonnage et enseignement & 245 \\
\hline \multicolumn{2}{|l|}{$2^{\mathrm{e}}$ section (industrielle) } \\
\hline Gammagraphie & 302 \\
\hline Irradiateurs & 52 \\
\hline Mesure de densité et pesage & 635 \\
\hline Mesure d'épaisseur & 900 \\
\hline Mesure de niveau & 705 \\
\hline Mesure d'humidité & 186 \\
\hline Mesure d'humidité + densité & 331 \\
\hline Elimination d'électricité statique & 60 \\
\hline Chromatographie en phase gazeuse & 536 \\
\hline Analyses diverses & 403 \\
\hline Etalonnage et enseignement & 567 \\
\hline
\end{tabular}

$N B$ : une autorisation peut couvrir plusieurs types d'utilisation. 
H. VIDAL

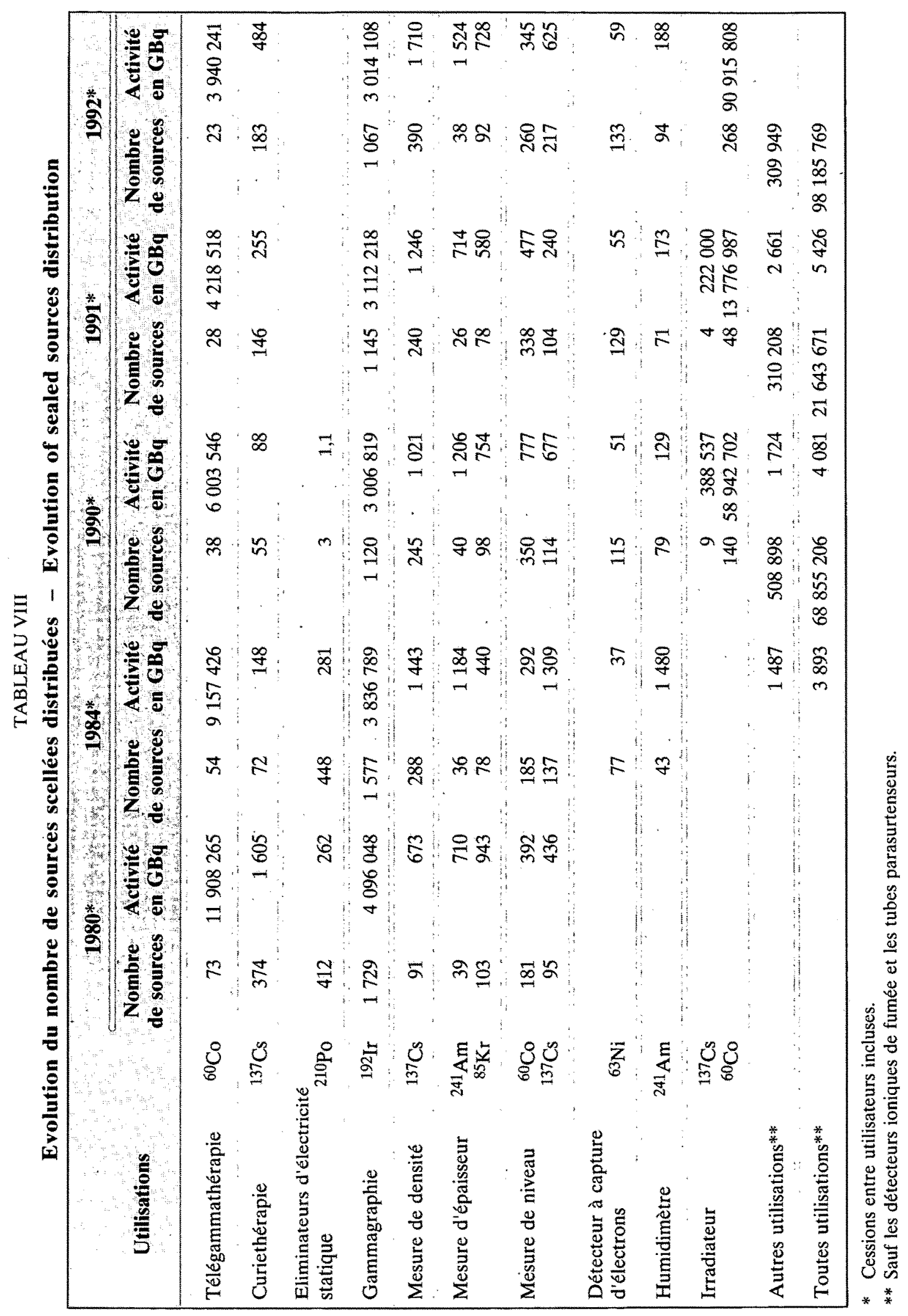


TABLEAU IX

Consommation des radioéléments (REA) en sources non scellées pour l'année 1992 (activité supérieure à $1 \mathrm{GBq}$ )

Unsealed source consumption in medicine in 1992 (activity over $1 \mathrm{GBq}$ )

Administrés à l'homme (thérapeutique et diagnostic)

\begin{tabular}{|lrrrr|}
\hline \multicolumn{1}{|c}{$\mathbf{R E A}$} & Nb. liv. & Nb. fdf & GBq & Activité en \\
& & & & mCi \\
${ }^{99} \mathrm{Tc}$ & 13456 & 1408 & 290041 & 7838961 \\
${ }^{133} \mathrm{Xe}$ & 2714 & 563 & 19559 & 528627 \\
${ }^{131} \mathbf{I}$ & 10852 & 3883 & 19240 & 520006 \\
${ }^{201} \mathrm{Tl}$ & 14001 & 5192 & 9926 & 268294 \\
${ }^{123} \mathrm{I}$ & 7422 & 1538 & 583 & 15765 \\
${ }^{67} \mathrm{Ga}$ & 3147 & 2152 & 497 & 13440 \\
${ }^{90} \mathrm{Y}$ & 655 & 498 & 283 & 7666 \\
${ }^{111} \mathrm{In}$ & 2855 & 1493 & 228 & 6179 \\
${ }^{186} \mathrm{Re}$ & 691 & 485 & 220 & 5951 \\
${ }^{32} \mathrm{P}$ & 500 & 356 & 171 & 4622 \\
${ }^{169} \mathrm{Er}$ & 581 & 435 & 104 & 2815 \\
${ }^{51} \mathrm{Cr}$ & 853 & 470 & 65 & 1764 \\
${ }^{125} \mathrm{I}$ & 483 & 226 & 18 & 504 \\
${ }^{81} \mathrm{Kr}$ & 35 & 3 & 15 & 420 \\
${ }^{192} \mathrm{Ir}$ & 7 & 3 & 6 & 163 \\
${ }^{85} \mathrm{Sr}$ & 10 & 10 & 3 & 83 \\
${ }^{89} \mathrm{Sr}$ & 16 & 15 & 2 & 64 \\
${ }^{59} \mathrm{Fe}$ & 109 & 77 & 2 & 55 \\
${ }^{57} \mathrm{Co}$ & 387 & 340 & 1,9 & 53 \\
\hline
\end{tabular}

Utilisés en diagnostic in vitro

\begin{tabular}{|c|c|c|c|c|}
\hline rar & Nb, live & $\mathbf{N b}$ fdf & 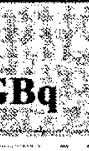 & $\operatorname{mej}^{4}$ \\
\hline${ }^{99} \mathrm{Tc}$ & 13 & 1 & 739 & 19994 \\
\hline $125 I$ & 74710 & 27810 & 134 & 3629 \\
\hline${ }^{14} \mathrm{C}$ & 489 & .236 & 13 & 363 \\
\hline${ }^{51} \mathrm{Cr}$ & 47 & 33 & 9 & 248 \\
\hline${ }^{3} \mathrm{H}$ & 2343 & 1178 & 8 & 222 \\
\hline $32 \mathbf{P}$ & 171 & 43 & 3 & 88 \\
\hline${ }^{35} \mathrm{~S}$ & 8 & 7 & 1 & 39 \\
\hline
\end{tabular}

Utilisés en curiethérapie

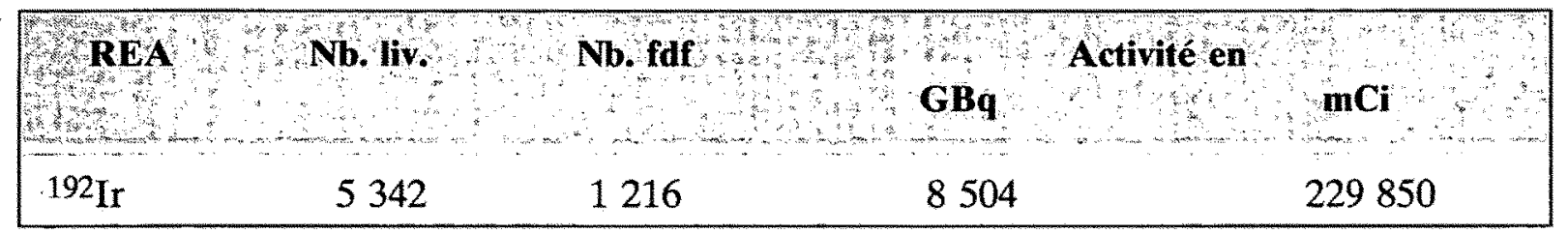

Nota $: \mathrm{Nb} . \mathrm{fdf}=$ nombre de formulaires de fourniture $; \mathrm{Nb}$. liv. = nombre de livraisons. 
H. VIDAL

TABLEAU $X$

Consommation des radioéléments en sources non scellées pour l'année 1992 (activité supérieure à $1 \mathrm{GBq}$ )

Unsealed source consumption (all uses) in 1992 (activity over 1 GBq)

Ensemble des utilisations

\begin{tabular}{|c|c|c|c|c|}
\hline REA & Nb. liv. & Nb. fdf & GBq & $\mathbf{m C i}$ \\
\hline${ }^{140} \mathrm{La}$ & 47 & 7 & 369960 & 9998919 \\
\hline${ }^{99} \mathrm{Tc}$ & 13581 & 1431 & 290806 & 7859631 \\
\hline${ }^{3} \mathrm{H}$ & 9562 & 6925 & 19918 & 538345 \\
\hline${ }^{133} \mathrm{Xe}$ & 2720 & 569 & 19560 & 528669 \\
\hline${ }^{131} I$ & 11184 & 4108 & 19449 & 525655 \\
\hline $201 \mathrm{~T} 1$ & 14015 & 5206 & 9929 & 268361 \\
\hline${ }^{192} \mathrm{Ir}$ & 5356 & 1226 & 8524 & 230393 \\
\hline${ }^{64} \mathrm{Cu}$ & 31 & 19 & 4144 & 112003 \\
\hline $125 \mathrm{I}$ & 8834 & 36760 & 1641 & 44375 \\
\hline${ }^{32} \mathrm{P}$ & 23602 & 8405 & 931 & 25182 \\
\hline $123 \mathrm{I}$ & 7532 & 1564 & 595 & 16094 \\
\hline${ }^{82} \mathrm{Kr}$ & 158 & 103 & 569 & 15388 \\
\hline${ }^{67} \mathrm{Ga}$ & 3154 & 2159 & 497 & 13455 \\
\hline${ }^{35} \mathrm{~S}$ & 6737 & 3938 & $453^{\circ}$ & 12260 \\
\hline${ }^{14} \mathrm{C}$ & 3615 & 3171 & 340 & 9193 \\
\hline${ }^{90} \mathrm{Y}$ & 658 & 500 & 285 & 7707 \\
\hline${ }^{111}$ In & 3029 & 1577 & 244 & 6606 \\
\hline${ }^{153} \mathrm{Sm}$ & 57 & 24 & 235 & 6355 \\
\hline${ }^{186} \mathrm{Re}$ & - 694 & 487 & 222 & 6002 \\
\hline${ }^{51} \mathrm{Cr}$ & 1888 & 1002 & 219 & 5936 \\
\hline${ }^{24} \mathrm{Na}$ & 16 & 7 & 148 & 4012 \\
\hline${ }^{169} \mathrm{Er}$ & 583 & 436 & 105 & 2839 \\
\hline${ }^{198} \mathrm{Au}$ & 4 & 4 & 79 & 2140 \\
\hline${ }^{241} \mathrm{Am}$ & 69 & 56 & 50 & 1353 \\
\hline${ }^{113} \mathrm{Sn}$ & 15 & 15 & 37 & 1005 \\
\hline${ }^{244} \mathrm{Cm}$ & 1 & 1 & 30 & 810 \\
\hline${ }^{81} \mathrm{Kr}$ & 35 & 3 & 15 & 420 \\
\hline${ }^{33} \mathrm{P}$ & 325 & 304 & 12 & 345 \\
\hline${ }^{45} \mathrm{Ca}$ & 178 & 127 & 12 & 344 \\
\hline${ }^{54} \mathrm{Mn}$ & 19 & 10 & 8 & 221 \\
\hline${ }^{177} \mathrm{Lu}$ & 3 & 3 & 7 & 200 \\
\hline${ }^{28} \mathrm{Al}$ & 37 & 9 & 6 & 162 \\
\hline${ }^{82} \mathrm{Br}$ & 3 & 2 & 5,9 & 160 \\
\hline${ }^{95} \mathrm{Zr}$ & 15 & 3 & 5,5 & 150 \\
\hline${ }^{86} \mathrm{Rb}$ & 58 & 56 & 3 & 89 \\
\hline${ }^{85} \mathrm{Sr}$ & 17 & 16 & 3 & 85 \\
\hline${ }^{59} \mathrm{Fe}$ & 145 & 102 & 2,8 & 76 \\
\hline${ }^{89} \mathrm{Sr}$ & 18 & 17 & 2 & 64 \\
\hline${ }^{57} \mathrm{Co}$ & 499 & 439 & 2 & 61 \\
\hline${ }^{65} \mathrm{Zn}$ & 25 & 20 & 1,7 & 47 \\
\hline${ }^{152} \mathrm{Eu}$ & 78 & 43 & 1 & 31 \\
\hline${ }^{233} \mathrm{U}$ & 2 & 1 & 1 & 30 \\
\hline
\end{tabular}

Nota $: \mathrm{Nb} . \mathrm{fdf}=$ nombre de formulaires de fourniture $; \mathrm{Nb}$. liv,$=$ nombre de livraisons. 


\section{Conclusion}

Dans cet article, on s'est efforcé de montrer que l'utilisation des rayonnements ionisants s'est tellement imposée dans tous les secteurs d'activité humaine tant médicaux qu'industriels, qu'il serait aujourd'hui impossible de s'en passer.

Des contraintes d'emploi deviennent, cependant, et fort heureusement, de plus en plus lourdes de façon que les risques associés soient minimisés à l'extrême.

\footnotetext{
N.B. : La Commission interministérielle des radioéléments artificiels (CIREA) a pour mission de formuler son avis ou ses propositions sur les questions d'ordre général que soulèvent l'élaboration ou l'application de la réglementation relative aux radioéléments artificiels (préparation, importation, fabrication, détention, transport, cession, radioprotection,...).

La Commission, présidée par un conseiller d'Etat, comprend des représentants des ministères intéressés (agriculture, défense, environnement, industrie, intérieur, santé, travail, universités) et de grands organismes concernés (Agence du médicament, CEA, CNRS, INSERM, SCPRI) ainsi qu'un secrétaire permanent. Les membres sont nommés par le Premier ministre.

Les autorisations concernant les activités liées aux applications à l'homme ou à la biologie humaine sont accordées par le ministre de la santé. Les autorisations concernant toutes les autres activités sont accordées par le président ou le secrétaire permanent de la Commission.
} 